\title{
Evolution of non-collinear magnetic state of exchange biased ferromagnet/normal metal/ ferromagnet/superconductor heterostructure in magnetic field studied by polarized neutron reflectometry
}

\author{
Yu. Khaydukov ${ }^{1,2,5}$, R. Morari ${ }^{3}$, V. Zdravkov ${ }^{3,4}$, L. Mustafa ${ }^{1}$, T. Keller $^{1,2}$, \\ B. Keimer ${ }^{1}$, and A. Sidorenko ${ }^{3}$ \\ ${ }^{1}$ Max-Planck-Institut für Festkörperforschung, Stuttgart, Germany \\ ${ }^{2}$ Max Planck Society Outstation at the FRM-II, Garching, Germany \\ ${ }^{3}$ D. Ghitsu Institute of Electronic Engineering and Nanotechnologies ASM, Kishinev, Moldova \\ E-mail: anatoli.sidorenko@kit.edu \\ ${ }^{4}$ Institut für Physik, Universität Augsburg, Augsburg, Germany \\ ${ }^{5}$ Skobeltsyn Institute of Nuclear Physics, Moscow State University, Moscow, Russia
}

Received January 2, 2017, published online May 25, 2017

\begin{abstract}
By using waveguide enhanced polarized neutron reflectometry we have characterized the magnetic state of exchange biased $\mathrm{CoO}_{x}(20 \mathrm{~nm}) / \mathrm{Co}(4 \mathrm{~nm}) / \mathrm{Nb}(5 \mathrm{~nm}) / \mathrm{Co}(2 \mathrm{~nm}) / \mathrm{Nb}(25 \mathrm{~nm}) / \mathrm{Al}_{2} \mathrm{O}_{3}$ system. Measurement allowed to determine the dependence of the inclination angles of magnetic moment of the both Co layers as a function of applied field. According to the measurement the soft $\operatorname{Co}(2 \mathrm{~nm})$ layer magnetization turns towards external field in magnetic fields as small as 20 Oe. In contrast direction of magnetic moment of Co(4 nm) layer can not be altered in magnetic fields as high as $2 \mathrm{kOe}$.
\end{abstract}

PACS: 75.70.Cn Magnetic properties of interfaces (multilayers, superlattices, heterostructures);

75.25.-j Spin arrangements in magnetically ordered materials (including neutron and spin-polarized electron studies, synchrotron-source $x$-ray scattering, etc.);

61.05.fj Neutron reflectometry.

Keywords: superconductor-ferromagnet, Polarized Neutron Reflectometry, triplet pairing.

Superconductor-feromagnet (SF) heterostructures are in the focus of current investigations, and reveal in the last years several experimental findings at nanoscale, based on development of unconventional correlations of superconducting pairs [1]. Quasi-one-dimensional Fulde-FerrellLarkin-Ovchinnikov (FFLO) superconducting state describes oscillating in space superconducting order parameter and related phenomena [2-4]. The appearance of the component with spin triplet symmetry in superconducting condensate is the base of another group of effects [5-8] and is favored by the presence of inhomogeneous, noncollinear magnetization. The spin polarized superconducting currents may flow in these systems and are of especial interest of superconducting spintronic $[9,10]$. The understanding of underlying physics and reliable control over experimentally measurable superconducting properties in
SF layered heterostructures is based on possibility of unambiguous determination of the value and magnetization directions in F-layers.

For the direct control of non-collinear magnetic state of SF structures polarized neutron reflectometry (PNR) can be used [11,12]. In PNR, the intensities of specularly reflected neutron beams with different polarization $R^{\mu \eta}(Q)$ are measured. Here $Q=4 \pi \sin (\theta) / \lambda$ is the momentum transfer, and $\theta$ and $\lambda$ are the grazing-incidence angle and the neutron wavelength, respectively. The indices $\mu$ and $\eta$ take values «+» or «-» and correspond to the projection of the neutron spin parallel to $H$ before and after the scattering process, respectively. The non-spin-flip (NSF) reflectivities $R^{++}$and $R^{-}$are sensitive to the depth profiles of sum and difference of nuclear scattering length density (SLD) and collinear component of the magnetization: 
$R^{ \pm \pm} \sim \rho_{0}(z) \pm M(z) \cos \alpha(z)$. Here $\rho_{0}(z), M(z)$ and $\alpha(z)$ are the depth profiles of nuclear SLD, absolute magnetization and angle between vectors of $\mathbf{M}$ and $\mathbf{H}$. In order to separate magnetic contribution the so-called spin asymmetry $S \equiv\left(R^{++}-R^{-}\right) /\left(R^{++}+R^{-}\right)$is often used in the analysis of the PNR data. It is easy to show that spin asymmetry is proportional to the depth profile of collinear component of magnetization: $S \sim M(z) \cos \alpha(z)$. The non-collinear component of magnetization causes the spin-flip scattering, which intensity is proportional to the noncollinear part of magnetization: $R^{+-} \approx R^{-+} \sim M(z) \sin \alpha(z)$. Thus simultaneous analysis of non-spin-flip and spin-flip channels allows the determination of depth profiles of the vector magnetization, and the experimental measurement of the level of magnetic non-collinearity. In this work we give one experimental example bases on the study of $\mathrm{Co} / \mathrm{Nb} / \mathrm{Co} / \mathrm{Nb}$ heterostructure.

Samples were prepared using magnetron sputtering system Leybold Z-400 (with a residual pressure in the cham-

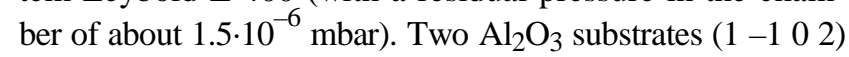
with areas $20 \times 20 \mathrm{~mm}$ and $5 \times 5 \mathrm{~mm}$ were simultaneously placed for samples deposition for further PNR and SQUID measurements. In total two targets were used for sputtering: first, the pure niobium (99.99\%) as a superconducting material for superconducting $\mathrm{Nb}$ layers deposition and ultra thin nonsuperconducting $\mathrm{Nb}$ films (less $5 \mathrm{~nm}$, as a interlayer metallic non superconducting spacer); second, the cobalt (99.9\%) for deposition of the ferromagnetic layers and for growing antiferromagnetic $\mathrm{CoO}_{x}$ films in atmosphere of mixture of $\mathrm{Ar}$ (purity 99.9995\%) and $\mathrm{O}_{2}$ (purity 99.9999\%) gazes.

Sputtering of $\mathrm{Nb}$ and Co layers was done in argon atmosphere of $8 \cdot 10^{-3}$ mbar pressure. In order to remove contaminations, such as absorbed gases and oxides, the substrates were etched and the targets were pre-sputtered for $3-5$ min before deposition of the multilayer structures. The deposition rates of the layers were: $3.5 \mathrm{~nm} / \mathrm{s}$ for $\mathrm{Nb}$, $0.5 \mathrm{~nm} / \mathrm{s}$ for pure Co film, and $0.1 \mathrm{~nm} / \mathrm{s}$ for $\mathrm{CoO}_{x}$.

The stack sequence was as follows: first the $\mathrm{Nb}(25 \mathrm{~nm})$ was deposited on the top of cleaned and etched substrates. Second layer $\operatorname{Co}(2 \mathrm{~nm})$ further named as Co1, than nonsuperconducting $\mathrm{Nb}(5 \mathrm{~nm})$ spacer, than second $\mathrm{Co}(4 \mathrm{~nm})$ further named as Co2. As a final step, the $\mathrm{CoO}_{x}(20 \mathrm{~nm})$ film was deposited in $\mathrm{Ar}: \mathrm{O}_{2}$ (4:1) mixture gases atmosphere with a summary pressure of $10^{-2}$ mbar. The ferromagnetic sub-system contains two ferromagnetic layers. The Co2 layer is supposed to be biased to the cobalt oxide film and did not change it's magnetization direction during the change of magnetic field orientation. The Co1 layer, in contrast is expected to be soft and change it's magnetization direction in relatively small magnetic fields. Similar system was recently used for the generation of triplet superconductivity [13].

Figure 1 shows the hysteresis loops measured at $T=300 \mathrm{~K}$ and at $T=13 \mathrm{~K}$ after cooling in magnetic field
$H=-5 \mathrm{kOe}$. The room temperature hysteresis loop consist of two hysteresis loops with coercive fields $H_{C 1} \approx 20$ Oe and $H_{C 2} \approx 250$ Oe which demonstrate different magnetic hardness of the Co1 and Co2 layers. After cooling of the sample in magnetic field $H=-5 \mathrm{kOe}$ the hysteresis loop changed significantly. Only one loop completely shifted down is seen. The last can be explained by the fact, that $\mathrm{Co} 2$ layer is biased to $\mathrm{CoO}_{x}$ layer and the magnetic field of $\pm 2 \mathrm{kOe}$ used for cycling is not enough to un-stick them.

In order to characterize quantitatively the non-collinear magnetic state of the structure at low temperatures we have performed PNR experiment. The PNR experiment was conducted at the angle-dispersive reflectometer NREX at the research reactor FRM-II in Garching, Germany. Polarized neutron beam with $\lambda=4.26 \pm 0.06 \AA$ and polarization of $99.99 \%$ falls on the sample under grazing incidence angles $\theta=\left[0.1-2^{\circ}\right]$. The divergence of the beam was set to $\Delta \theta=0.03$ by two slits before the sample. The polarization of the reflected beam was analyzed by a polarization analyzer with the efficiency of $99.10 \%$.The sample cooled in magnetic field of $H=-5 \mathrm{kOe}$ down to $T=7 \mathrm{~K}$, which is above superconducting transition temperature of $\mathrm{Nb}$ (see inset in Fig. 1). To make a non-collinear magnetic state the following procedure was used. At $T=7 \mathrm{~K}$ the field was released to zero and sample was rotated on $90^{\circ}$ along $z$-axis (see Fig. 2(d)). After that, the reflectivity curves were measured in four spin channels in magnetic fields $H=6,16$, 23, 36, 50 and 100 Oe. Figure 2(a) shows the reflectivity curves measured in $H=100$ Oe. On the graph the total reflection plateau is seen on the NSF curves $R^{++}$and $R^{--}$for $Q<Q_{\text {crit }}=0.16 \mathrm{~nm}^{-1}$ caused by the reflection from thick $\mathrm{Al}_{2} \mathrm{O}_{3}$ substrate.

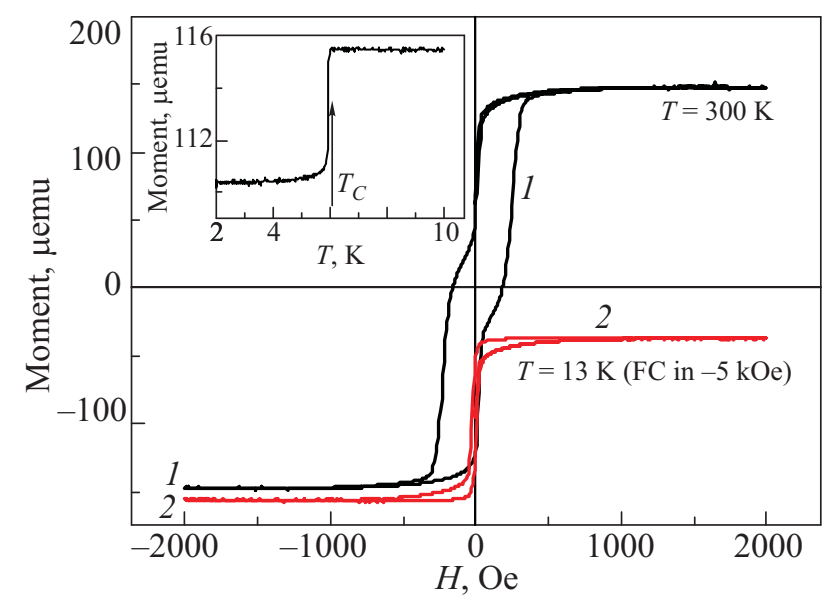

Fig. 1. (Color online) The hysteresis loop measured at room temperature (black curve 1 ) and at $T=13 \mathrm{~K}$ after cooling the $\mathrm{Nb} / \mathrm{Co} 1 / \mathrm{Nb}$ (thin)/Co2/CoO ${ }_{x}$ sample in $H=-5 \mathrm{kOe}$ (red curve 2). Inset shows the temperature dependence of the magnetic moment at $H=50$ Oe at low temperatures. Decrease of the magnetic moment below $T_{C}=6 \mathrm{~K}$ is caused by transition of Nb layer to superconducting state. 


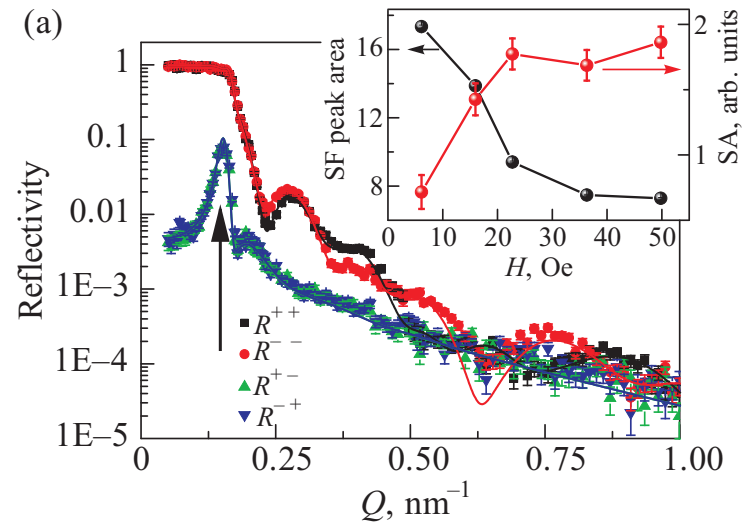

(b)

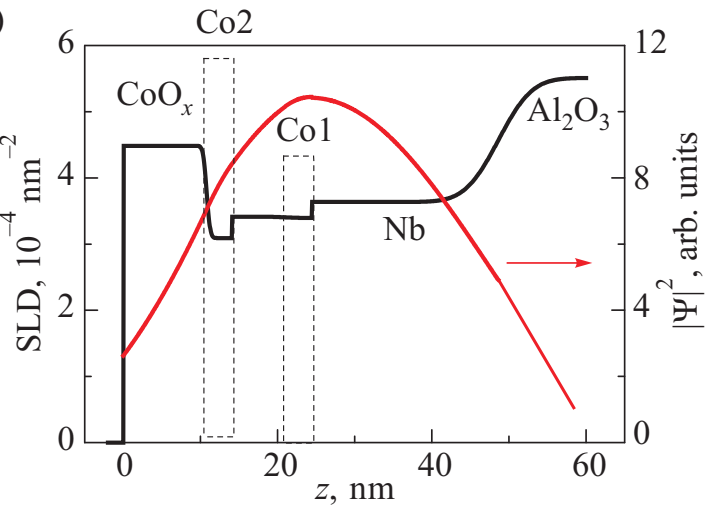

(c)

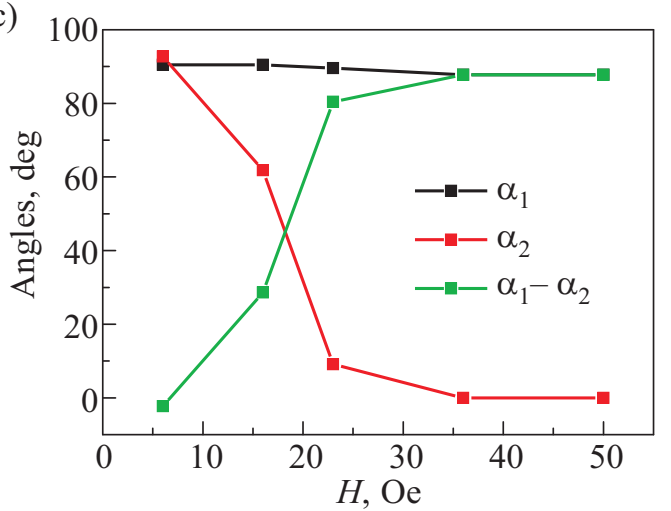

(d)

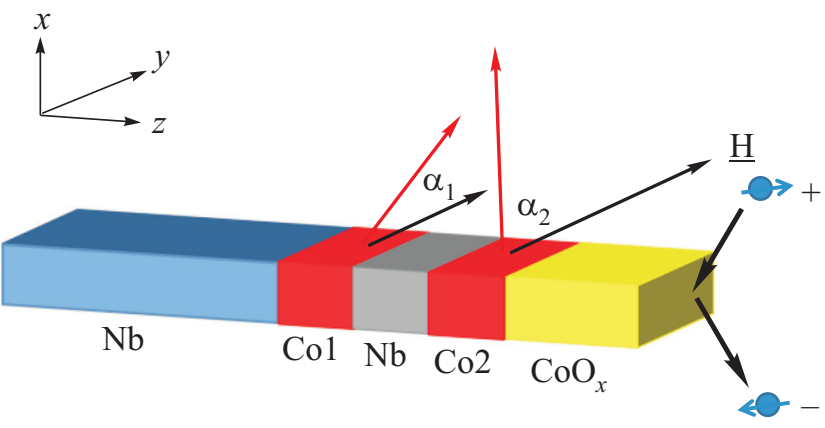

Fig. 2. (Color online) Polarized neutron reflectometry data. (a) Experimental (dots) and model (lines) reflectivity curves measured at $T=7 \mathrm{~K}$ and $H=100 \mathrm{Oe}$. The vertical arrow shows the position of waveguide enhancement. Inset: Field evolution of the spin-flip peak area (black dots) and integrated spin asymmetry (red dots). (b) The depth profiles of nuclear SLD (black line) and neutron density at resonance $Q$. (c) The field evolution of inclination angles $\alpha_{1}$ and $\alpha_{2}$ of magnetic layers (black and red dots correspondingly) and relative angle $\alpha_{1-2} \equiv \alpha_{1}-\alpha_{2}$. (d) The sketch of the PNR experiment and sample Red arrows show direction of magnetization in Co layers.

At $Q>Q_{\text {crit }}$ neutrons start to penetrate inside the structure, reflect from different interfaces with different amplitudes and phases and as a sequence, interfere, forming the so-called Kiessig fringes. The observed on experiment difference between $R^{++}$and $R^{-}$shows the presence of collinear component of magnetization. In addition to NSF curves we have observed strong spin-flip scattering with the peak of amplitude of $10 \%$ positioned close to $Q_{\text {crit }}$. The peak itself is caused by the so-called waveguide enhancement $[13,14]$, which will be explained below.

Thus presence of both spin-flip scattering and spin asymmetry at $H=100$ Oe evidences the presence of both collinear and non-collinear components of magnetization in the structure. Inset in Fig. 2(a) shows the field evolution of integrated spin-flip scattering and spin asymmetry. One can see that system is characterized by two states. First, at $H \rightarrow 0$ strong spin flip scattering and absence of spin asymmetry can be seen. This means that all magnetic moments in the system are aligned non-collinearly to the direction of external field. At further increase of external field above 20 Oe intensity of spin-flip scattering decreases (but not disappears) and spin asymmetry appears. This can be interpreted as presence of both collinear and non-collinear magnetic moments in the system. In order to describe quantitatively these two states we have fitted experimental data to the model curves varying structural parameters and magnetic parameters of the layers. The free structural parameters were thicknesses of the layers, their SLD and rms roughness of the interfaces. The magnetic depth profile was parametrized using absolute values of magnetization $\left(M_{1}\right.$ and $\left.M_{2}\right)$ and angles of misalignment $\left(\alpha_{1}\right.$ and $\left.\alpha_{2}\right)$ of $\mathrm{Co} 1$ and Co2 layers. The best-fit model curves are shown in Fig. 2(a) by the solid lines. One can see from Fig. 2(a) a very good agreement between theory and model. The resulted nuclear SLD depth profile is shown in Fig. 2(b). Due to the high scattering length of oxygen atoms layers of $\mathrm{CoO}_{x}$ and $\mathrm{Al}_{2} \mathrm{O}_{3}$ have higher SLD than Co and Nb layers between them. As a result, the SLD depth profile forms well-like structure which allows to "trap" neutrons at certain $Q \approx Q_{\text {crit }}$ and thus enhance neutron density inside the structure by factor of 10 (see red line in Fig. 2(b)). As a result spin-flip scattering is also enhanced at this $Q$ increasing thus sensitivity to the non-collinear component of magnetization [13,14]. Vector of the magnetic state at this field can be described by $\alpha_{1}=0^{\circ}$ and $\alpha_{2}=89^{\circ}$, i.e., Co1 layer already flipped at $H=100$ Oe towards direction of external field while $\mathrm{Co} 2$ is still biased to $\mathrm{CoO}_{x}$ layer. The data obtained at other fields were treated in similar way. Fig. 2(c) shows 
the field evolution of angles $\alpha_{1}$ and $\alpha_{2}$ and their difference $\Delta \alpha$. As it follows from Fig. 2(c) magnetic state at $H=0$ is characterized by both Co layers which magnetic moments are parallel to each other and normal to the external field. After applying $H>20$ Oe the soft Co1 layer is flipped towards external field while Co2 magnetization is still normal to the direction of external field.

Thus conducted PNR experiment on $\mathrm{Nb} / \mathrm{Co} 1 / \mathrm{Nb} / \mathrm{Co} 2 / \mathrm{CoO}_{x}$ multilayered structure allowed to measure directly and separately vector of magnetic moments of both Co layers and trace their evolution with changes of magnetic field.

This work was carried out in the framework of a collaboration agreement between Max-Planck-Institut für Festkörperforschung and Institute of Electronic Engineering and Nanotechnologies ASM, and based upon experiments performed at the NREX instrument operated by Max-Planck Society at the Heinz Maier-Leibnitz Zentrum (MLZ), Garching, Germany. The neutron part of the project was supported by the European Commission under the 7th Framework Programme through the "Research Infrastructures" action of the Capacities Programme, NMI3-II, Grant Agreement number 283883, and by the project STCU\#5982.

1. M.G. Blamire and J.W.A. Robinson, J. Phys.: Condens. Matter 26, 453201 (2014).

2. A.I. Buzdin, Rev. Mod. Phys. 77, 935 (2005).

3. V.V. Ryazanov, V.A. Oboznov, A.Yu. Rusanov, A.V. Veretennikov, A.A. Golubov, and J. Aarts, Phys. Rev. Lett. 86, 2427 (2001).
4. V. Zdravkov, A. Sidorenko, G. Obermeier, S. Gsell, M. Schreck, C. Müller, S. Horn, R. Tidecks, and L.R. Tagirov, Phys. Rev. Lett. 97, 057004 (2006).

5. F.S. Bergeret, A.F. Volkov, and K.B. Efetov, Rev. Mod. Phys. 77, 1321 (2005).

6. Trupti S. Khaire, Mazin A. Khasawneh, W.P. Pratt, Jr., and Norman O. Birge, Phys. Rev. Lett. 104, 137002 (2010).

7. P.V. Leksin, N.N. Garif'yanov, I.A. Garifullin, Ya.V. Fominov, J. Schumann, Y. Krupskaya, V. Kataev, O.G. Schmidt, and B. Büchner, Phys. Rev. Lett. 109, 057005 (2012).

8. V.I. Zdravkov, J. Kehrle, G. Obermeier, D. Lenk, H.-A. Krug von Nidda, C. Müller, M.Yu. Kupriyanov, A.S. Sidorenko, S. Horn, R. Tidecks, and L.R. Tagirov, Phys. Rev. B 87, 144507 (2013).

9. J. Linder and J.W.A. Robinson, Nature Phys. 11, 307 (2015).

10. M. Eschrig, Phys. Today 64, 43 (2011).

11. C. Klose, T.S. Khaire, Y. Wang, W.P. Pratt, N.O. Birge, B.J. McMorran, T.P. Ginley, J.A. Borchers, B.J. Kirby, B.B. Maranville, and J. Unguris, Phys. Rev. Lett. 108, 127002 (2012).

12. Yu.N. Khaydukov, G.A. Ovsyannikov, A.E. Sheyerman, K.Y. Constantinian, L. Mustafa, T. Keller, M.A. Uribe-Laverde, Yu.V. Kislinskii, A.V. Shadrin, A. Kalaboukhov, B. Keimer, and D. Winkler, Phys. Rev. B 90, 035130 (2014).

13. M.G. Flokstra, T.C. Cunningham, J. Kim, N. Satchell, G. Burnell, P.J. Curran, S.J. Bending, C.J. Kinane, J.F.K. Cooper, S. Langridge, A. Isidori, N. Pugach, M. Eschrig, and S.L. Lee, Phys. Rev. B 91, 060501(R) (2015).

14. Yu.N. Khaydukov, Yu.V. Nikitenko, L. Bottyan, A. Rühm, and V.L. Aksenov, Cryst. Rep. 55, 1235 (2010).

15. Yu.N. Khaidukov and Yu.V. Nikitenko, Nucl. Instum. Meth. A 629, 245 (2011). 